\title{
Directional tunneling escape from nearly spherical optical resonators
}

\author{
Scott Lacey, Hailin Wang, David H. Foster and Jens U. Nöcke* \\ Department of Physics, University of Oregon, Eugene, OR 97403
}

(Dated: 07/17/2003, Phys. Rev. Lett. 91, 033902 (2003))

\begin{abstract}
We report the surprising observation of directional tunneling escape from nearly spherical fused silica optical resonators, in which most of the phase space is filled with non-chaotic regular trajectories. Experimental and theoretical studies of the dependence of the far-field emission pattern on both the degree of deformation and the excitation condition show that non-perturbative phase space structures in the internal ray dynamics profoundly affect tunneling leakage of the whispering gallery modes.
\end{abstract}

Electromagnetic fields in a uniform dielectric sphere can be calculated in much the same way as quantum mechanical wave functions in a spherically symmetric potential. The sphere exhibits whispering-gallery (WG) modes, which are long-lived resonances with electromagnetic fields confined near the surface 1]. For small deviations from the spherical shape, $\epsilon \equiv\left(R_{\max }-\right.$ $\left.R_{\min }\right) /\left(R_{\max }+R_{\min }\right) \ll 1$ (where $R_{\max }, R_{\min }$ are the maximal and minimal radii), perturbative treatments [2] are routinely employed to infer deformation parameters from the splitting of azimuthal degeneracy of the WG modes [3]. Certain strongly non-spherical resonators, on the other hand, can be analyzed with methods from quantum chaos such as random-matrix theory or periodicorbit expansions [4]. Many generic resonator shapes, however, fall into a transition regime in which none of these known methods apply globally. When entering this regime from the perturbative side, calculations may encounter singularities and undefined limits [5]. Experimental studies in this regime can thus provide insight into how nature resolves the competition between perturbative and non-perturbative physics, here with the resonator shape as a control parameter.

In this paper we present studies of far-field emission patterns and resonance lifetimes of deformed fused-silica microspheres. The experimental and theoretical studies of the dependence of the far-field emission pattern on both the degree of deformation and the excitation condition show that highly directional tunneling escape can occur in microspheres with small deformation $(\epsilon \approx 1 \%)$ and with a large size parameter $(k R=2 \pi R / \lambda \approx 785$, where $\lambda \approx 800 \mathrm{~nm}, R \approx 100 \mu \mathrm{m})$. These results are completely unexpected in the ray optics limit or in the earlier perturbative wave treatment with $\epsilon$ as a small parameter. The observed emission pattern shows that on one hand, the ray model breaks down in predicting the escape mechanism of WG modes for resonators with small deformation. On the other hand, the nonperturbative phase space structures predicted by the ray model can profoundly affect tunneling leakage of the WG modes. Even at extremely small deformation, nonperturbative phase space structures still persist and are directly responsible for directional tunneling escape. This intricate interplay between ray and wave dynamics provides essential physical insights into properties of weakly deformed WG resonators, especially, for those in which the phase space is filled with non-chaotic regular trajectories. Directional emission patterns have previously been observed in more strongly deformed resonators where a significant fraction of the internal rays shows chaotic motion [6].

Deformed microspheres were formed by melting together two spheres of similar sizes. The individual spheres were fabricated by melting an optical fiber tip with a focused $\mathrm{CO}_{2}$ laser beam. The spheres were brought into contact and heated until surface tension produced a completely convex surface. By carefully controlling the temperature of the glass using the $\mathrm{CO}_{2}$ laser, it was possible to repeatedly reduce the degree of deformation. For reference, we define the elongated axis of the resulting prolate spheroid, which also connects the centers of the two original spheres, to be the $x$-axis. The $z$-axis is defined by the remaining fiber stem, which breaks the rotational symmetry about the $x$-axis, making the deformed microsphere completely non-axisymmetric. The stem was held by a fiber chuck for easy manipulation of the sphere. Images of these deformed microspheres taken from three orthogonal directions have been shown in an earlier study [7].

To investigate emission properties and resonance lifetimes of the deformed microspheres, we use frustrated total internal reflection at the surface of a prism with refractive index $n=1.7$ to launch individual travelingwave WG modes near the $x-y$ plane of the spheroid (Fig. [1. inset) [8]. The initial angle of incidence $\chi_{0}$ with which the WG modes are launched in the microsphere can be controlled by adjusting the angle of incidence $\psi$ in the prism.

WG mode spectra were obtained by measuring the far-field emission intensity as a function of the excitation wavelength. Since the stem holding the resonator is a strong leakage pathway, the observed long-lived WG modes must have internal field patterns that do not overlap with the stem region. This is made possible by the fact that the resonator is slightly flattened in the $z$ direction (along the stem axis). Experiments as well as ray simulations show that this deformation stabilizes the rays in the vicinity of the $x-y$ plane. Our direction- 


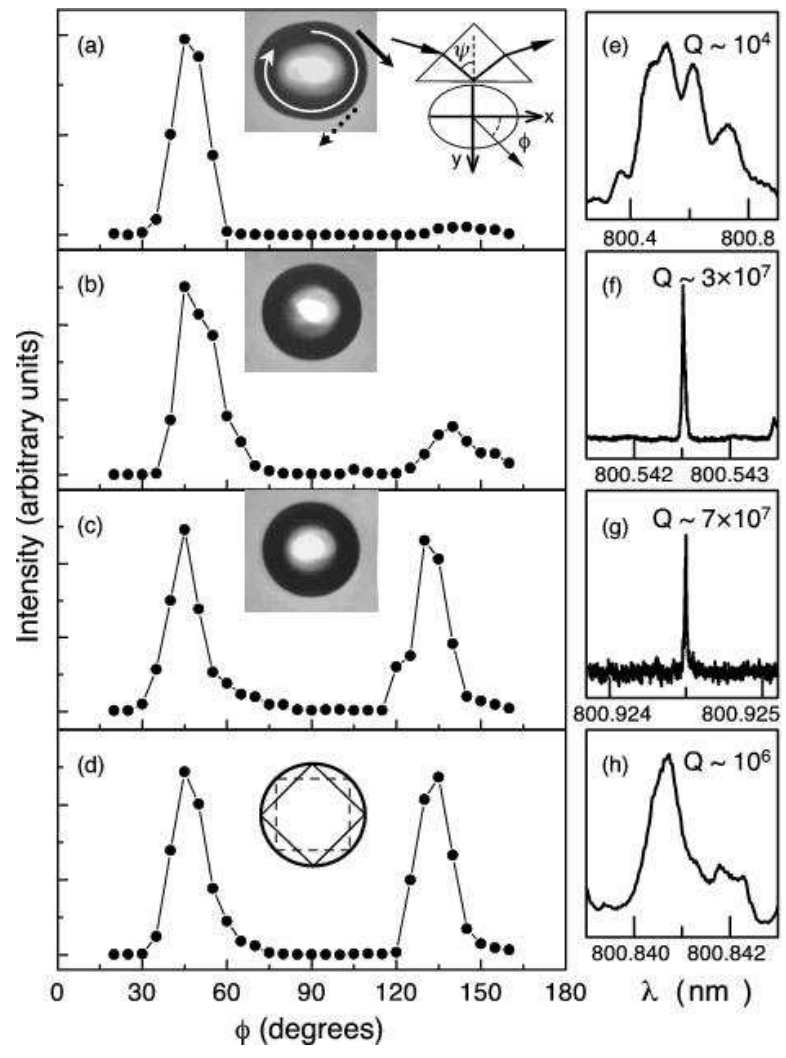

FIG. 1: (a)-(d): Far-field emission patterns of WG modes. Insets: bottom view of the resonators showing the progression of shapes in the $x-y$ cross-section, from which we determine $\epsilon=6.7 \%$ (a), $\epsilon=2.2 \%$ (b), and $\epsilon=1.2 \%$ (c,d). WG modes were launched at $\sin \chi_{0} \approx 1$ in (a)-(c), and at $\sin \chi_{0} \approx 0.8$ in $(\mathrm{d}) ; \chi$ is the internal angle of incidence with respect to the surface normal. (e)-(h): the spectra corresponding to the modes in (a)-(d), from which we deduce the Q-factors. Inset to (d): stable (solid) and unstable (dashed) 4-bounce orbits in a quadrupole at $\epsilon=1.2 \%$.

ality measurements correspondingly were performed in this plane. By scanning the detector while keeping the resonator fixed, we recorded WG mode spectra at various angles, $\phi$ (from the $x$-axis), to construct the farfield emission pattern at a given microsphere deformation $\epsilon$. The microsphere was then reheated to reduce $\epsilon$ and the far-field emission pattern was measured again, leading to the evolution shown in Figure 11 The excitation beam is s-polarized, and the detection scheme is polarization-insensitive. The far-field emission patterns are independent of the polarization of the excitation laser beam. Spheroids with similar $\epsilon$ exhibit qualitatively the same behavior in terms of mode spectra, Q-factors, and emission patterns.

Beginning with a strongly deformed microsphere, the emission was observed to have a strong peak at $\phi=45^{\circ}$ in the far field [see Fig. 1 (a)]. Since clockwise traveling waves were excited, as indicated by the white arrow in the sphere image in Fig. 1 (a), this far-field emission direction corresponds to light escaping tangential from the region at $\phi=-45^{\circ}$ on the surface of the microsphere, shown by the solid arrow in the sphere image. A much smaller peak ( $5 \%$ of the large peak height) in the farfield emission pattern was observed at $\phi=135^{\circ}$, corresponding to light escaping from the region at $\phi=45^{\circ}$ on the microsphere surface (dotted arrow). As the deformation was reduced, the far-field emission peak at $\phi=135^{\circ}$ grew to about one quarter of the $\phi=45^{\circ}$-peak height in Fig. 1 (b). In Fig. 10 (c), the two peaks reached nearly equal intensity. The bright emission spots at $\phi= \pm 45^{\circ}$ on the resonator surface can be viewed directly with a CCD camera. For reference, we call the pattern in Fig. [1 (a) asymmetric and the pattern in Fig. 10 (c) symmetric (around $\left.\phi=90^{\circ}\right)$. Figures $1(\mathrm{e})-(\mathrm{g})$ also show that as the deformation was reduced, the Q-factor of the relevant WG modes increased by nearly four orders of magnitude.

The measurements discussed thus far were performed with input condition $\chi_{0} \approx 90^{\circ}\left(\sin \chi_{0} \approx 1\right)$. Figure 1 (d) shows the far-field emission pattern from the same microsphere as Fig. 1 (c) but with light launched at $\sin \chi_{0} \approx 0.8$. The emission pattern is nearly identical to Fig 1 (c) where $\sin \chi_{0} \approx 1$, although the corresponding Q-factor is nearly two orders of magnitude smaller [Figs. 10 (g) and (h)].

The observed emission patterns in Fig. 10 (a)-(d) disagree with the intuitive expectation that WG modes in an oval resonator should preferentially emit tangential to the points of highest curvature, into the far-field direction $\phi= \pm 90^{\circ}$. This is what one obtains when modeling our spheroids as triaxial ellipsoids, whose internal ray dynamics exhibits no chaos [9], independently of the axis ratios.

The observed emission pattern of the most deformed microsphere, Fig. 10 (a), is well explained by a ray model. The peak at $\phi=45^{\circ}$ can be attributed to an effect known as dynamical eclipsing [6]. In the ray model, a WG mode corresponds to light rays trapped close to the perimeter of the dielectric resonator by total internal reflection, which prevents light escape unless a critical angle $\chi_{c} \equiv \arcsin 1 / n=43.6^{\circ}$ is reached, where $n=1.45$ is the refractive index of the fused silica. At small but finite $\epsilon$, any oval can be approximated by the first terms of a multipole expansion, which after proper choice of origin is quadrupolar. In this limit, a stable 4-bounce orbit shaped like a diamond forms with its sharp vertices at the highest-curvature points of the resonator with an angle of incidence $\chi_{4} \approx 45^{\circ}$ [inset to Fig. 1. (d)]. This creates "islands of stability" in phase space [4]: rays launched near the diamond orbit will retain similar reflection points and angles even if they do not close onto themselves after four bounces. At the deformation used in Fig. 囵(a), most of the phase space supporting the WG mode in question is chaotic. However, chaotic rays cannot penetrate the 4-bounce islands; this means chaotic WG rays are pre- 
vented from refractively escaping at the points of highest curvature, because $\chi_{4} \approx \chi_{c}$. Instead, as trajectories flow around the islands in one direction, refractive escape occurs near $\phi=-45^{\circ}$ on the resonator surface. From this circulation around the islands, asymmetric far-field emission patterns result as a hallmark of refractive escape. This is what we observe in Fig. 1 (a).

This ray mechanism was originally proposed and tested for effectively 2D systems where islands of stability and chaotic regions are mutually exclusive. In our 3D spheroids, the same diamond-shaped stable orbit exists in or near the $x-y$ plane, and refractively escaping rays in its vicinity have lifetimes that correspond to $\leq 10$ round trips in the resonator, translating to a Q-factor near $10^{4}$. During this time, a given ray can be considered as moving in a cross-sectional plane that may be inclined and slowly precessing around the $z$-axis. The variation of cross-sectional shape, $\Delta \epsilon$, probed by such rays causes no significant differences in the size of the 4-bounce islands [10]. Therefore, rays in the $3 \mathrm{D}$ resonator behave as in a corresponding fixed 2D resonator during a relatively short time preceding an escape event, and in particular show dynamical eclipsing.

However, refractive escape ceases to be the dominant leakage mechanism at the very small deformation used in Figs. 10 (c) and (d), since in this case chaotic diffusion is no longer effective and the stable diamond orbit itself also becomes fully confined by total internal reflection. Those classical rays that do escape refractively, moreover, are not able to produce the symmetric emission patterns observed in Figs. 1(c) and (d) 11]. Hence, the emission process in these cases is governed by evanescent escape, i.e. a tunnel coupling between internal and radiation fields.

Tunneling is the only decay mechanism in an ideal sphere where each WG mode in the plane of excitation corresponds to a unique azimuthal angular momentum number $m$ with respect to the $z$-axis, which is semiclassically related to $\chi_{0}$ by $\sin \chi_{0}=m /(n k R)$, using the fact that the modes of interest remain close to the $x-y$ plane and hence have total angular momentum $l \approx m$. At $k R \gg 1$, the tunneling escape rate is negligible when $\sin \chi_{0} \approx 1$ but accelerates exponentially as $\sin \chi_{0}$ approaches $\sin \chi_{c}$. In a deformed microsphere, the angle of incidence $\chi$ varies as a well-defined function of $\phi$ provided it covers an angular momentum range where chaos is neglibible 12. A circulating ray with varying $\chi(\phi)>\chi_{c}$ will then escape with exponentially strong selectivity near the minima of $\chi(\phi)$.

The emission locations and directions observed in the experiment indicate that the minima of $\chi(\phi)$ lie near the corners of the unstable rectangular 4-bounce orbit [inset to Fig. [1(d)]. We determined from ray tracing that this only occurs near but above $\chi_{c}$. Thus, the symmetric far-

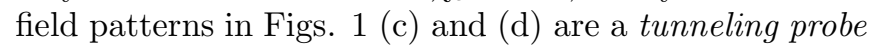
for the minima of the ray-optical $\chi(\phi)$ in the vicinity of the critical angle $\chi_{c}$. To further corroborate this interpretation, recall that the emission patterns as shown in Figs. 11 (c) and (d) are insensitive to the initial angle of incidence, $\chi_{0}$, as they should be if the detected light originates near $\sin \chi \approx \sin \chi_{c}$. The question remains how the light coupled into the resonator is able to reduce its angular momentum from a high value corresponding to $\sin \chi_{0}$ to the much lower $\sin \chi_{c}$. Our observation shows that such a dynamical process is present in the spheroid, but does not unambiguously reveal its mechanism. A possible mechanism is Arnol'd diffusion, a phase-space transport process that crucially depends on the fact that the resonators are in fact three-dimensional [7], with no axial symmetry. Even without resolving this question, we can nevertheless conclude that the phase space region near $\sin \chi_{c}$ is responsible for the observed emission patterns.

Our initial assumption of a 2D model for the emission process, justified by the approximate $2 \mathrm{D}$ nature of the $3 \mathrm{D}$ ray trajectories for intermediate times as discussed earlier, is therefore consistent with the fact that no longtime properties of the internal ray dynamics are needed to explain the emission directions. With the same justification, we also performed numerical wave calculations by modeling the shape felt by modes in the $x-y$ plane as the cross section of a quadrupolar cylinder. Figures 2 (a) and (b) plot the intensity patterns of two WG modes under traveling-wave excitation [12, 13. The calculation assumes s-polarized light and uses size parameters near $k R \approx 113$ [14].

The emission patterns in Figs. 2 (a) and (b) agree qualitatively with the asymmetric and the symmetric emission patterns shown in Figs. 1 (b) and (d), respectively. To understand the physical mechanism for the emission in Fig. 2 (b), we plot in Fig. 2 (c) the distribution of angular momentum numbers for the WG mode in (b). Figure2 (c) shows negligible overlap with the window for refractive escape below the critical angle $\chi_{c}$ (corresponding to a critical angular momentum $m_{c}=k R \approx 113$ ). This indicates that the emission from this mode is due to tunneling escape. By comparison, both refractive and tunneling escape contribute to the emission in Fig. 2 (a), with the refractive escape playing a dominant role.

The peak splitting of width $\Delta m \approx 4$ in Fig. 2 (c) is a straightforward consequence of the oval deformation: as the wave circulates around the resonator with varying radius between $R_{\min }$ and $R_{\max }$, its angular momentum oscillates but has high probabitity of being near its extrema, given by the extrema of $m \approx r(\phi) n k R \sin \chi(\phi)$ over the surface. The fact that the minima of $\chi(\phi)$ do not occur at $\phi=0^{\circ}, 180^{\circ}$ on the surface is due to the 4bounce island structure in the vicinity of $\sin \chi=\sin 45^{\circ}$.

For comparison, Fig. 2 (c) also shows the distribution of angular momentum number of an ultra-high $\mathrm{Q}$ WG mode with emission directionality as expected for the ellipse [see Fig. 22(d)]. The mode is confined at a 


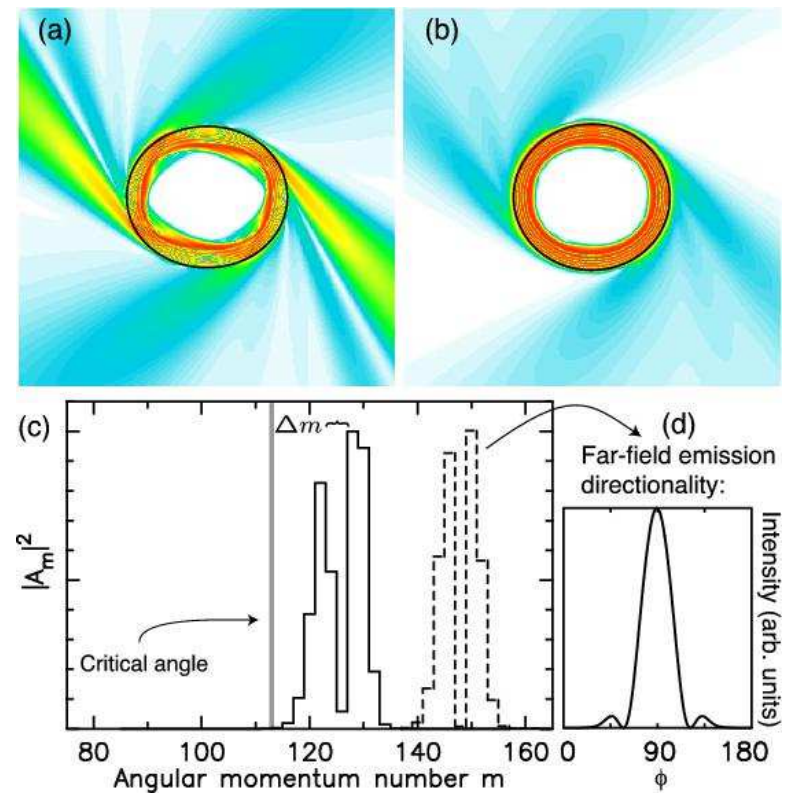

FIG. 2: Top: Intensity pattern of WG modes with parameters $k R=112.063, \epsilon=6.5 \%$ (a) and $k R=112.452, \epsilon=3.4 \%$ (b). The Q-factors are $4 \times 10^{4}$ (a) and $2 \times 10^{5}$ (b), and the overall directionality agrees with that in Fig. 1 (b) and (d), respectively. The values of $\epsilon$ differ from Fig. 1 because the simulation approximates the unknown details of the experimental shape by a pure quadrupole 12]. Bottom: solid histogram in (c) shows the angular-momentum distribution of the mode in (b). A very high-Q WG mode at the same deformation (c, dashed), with $k R=113.229$ and $Q=3 \times 10^{14}$ radiates predominantly into $\phi=90^{\circ}$, as shown in the far-field pattern (d).

high $m$, corresponding to $\sin \chi_{0} \approx 0.91$. Note that here the tunneling loss is negligible compared with scattering or absorption loss of the material that limits the actual Q-factor of fused silica microspheres to of order $10^{9}$ [15]. The two modes in Fig. 2(c) have practically no overlap in angular momentum space. The qualitative difference in the far-field patterns of these two modes further confirms that the mode shown in Fig. 11 (c), which was excited at $\sin \chi_{0} \approx 1$, is not confined to $\sin \chi_{0} \approx 1$.

In essence, our experiment exploits the peculiar feature that for fused silica the critical angle lies near the phase-space islands corresponding to the stable 4-bounce orbit. These islands are the dominant structure in the WG region and persist even at small $\epsilon$ where most of the phase space is filled with non-chaotic, "regular" trajectories. The island formation is a non-perturbative consequence of the breakdown of conservation laws [9], which qualitatively distinguishes the generic quadrupolar shape from an ideal ellipse even though the two shapes differ only to second order in $\epsilon$. The emission characteristics of a silica spheroid in the seemingly trivial small- $\epsilon$ regime will continue to be strongly affected by ray patterns that wrap around the perimeter in approximately four bounces.

Whether any non-perturbative structure in the ray dynamics can be resolved by the wave field, depends on $k R$ [16]: a directionality measurement will be able to distinguish the peaks at $\phi=90^{\circ} \pm 45^{\circ}$ if the conjugate angular momentum $m$ satisfies the uncertainty relation $\Delta \phi \Delta m \geq$ $1 / 2$ with $\Delta \phi \approx \pi / 4$. This implies $\Delta m>2 / \pi \approx 1$, which in our spheroids translates to a fluctuating angle of incidence of $\Delta \sin \chi=\Delta m /(n k R) \approx 10^{-3}$. Even the least deformed resonator $(\epsilon \approx 1 \%)$ exceeded this estimated resolution threshold significantly.

It is truly remarkable that this intricate interplay between ray and wave dynamics for which chaos plays no dominant role, can be exploited to engineer WG modes that can feature both high-Q and highly directional emission. This makes WG resonators with small deformation highly promising for a variety of applications 17, 18], such as microlasers, nonlinear optics, and quantum information processing.

This work was supported in part by NSF under grants No. DMR9733230 and No. DMR0201784.

* Electronic address: noeckel@uoregon.edu URL: http://oco.uoregon.edu

[1] L.G.Guimarães and H.M.Nussenzveig, J. Mod. Opt. 41, 625 (1994); G. Roll and G. Schweiger, J. Opt. Soc. Am. A 17, 1301 (2000).

[2] S. Ng, P. Leung, and K. Lee, J. Opt. Soc. Am. B 19, 154 (2002); and references therein.

[3] W. von Klitzing et al., Opt. Lett. 26, 166 (2001).

[4] H.-J. Stöckmann, Quantum Chaos, An Introduction (Cambridge University Press, 1999).

[5] M. V. Berry, Phys. Today 55 (May), No. 5, p. 10 (2002).

[6] S. Chang et al., J. Opt. Soc. Am. B 17, 1828 (2000).

[7] S. Lacey and H. L. Wang, Opt. Lett. 26, 1943 (2001).

[8] The measurement was performed with a tunable diode laser (New Focus).

[9] H. Waalkens, J. Wiersig, and H. R. Dullin, Ann. Phys. 276, 64 (1999).

[10] S. S. Chang, N. B. Rex, and R. K. Chang, J. Opt. Soc. Am. B 16, 1224 (1999).

[11] Even at small deformation, the separatrix region we are probing is created by the homoclinic tangle of the unstable, rectangle-shaped periodic orbit shown dashed in the inset to Fig. 1 (d), and this tangle exhibits an asymmetry which will produce asymmetric emission whenever the escape is predominantly ray-based.

[12] J. U. Nöckel and A. D. Stone, Nature 385, 45 (1997).

[13] I. Braun et al., Appl. Phys. B 70, 335 (2000).

[14] We have performed the same calculations at wave numbers $k R \approx 70$, and close to the current limits of our numerical technique at $k R \approx 200$. All these results are consistent with the ones presented in Fig. 2, showing that the phase-space structure causing the observed emission is already fully resolved in Fig. 2. Extrapolating to the experiment (Fig. 1 ) at $k R \approx 785$, we expect no further changes within the angular resolution of the detector ap- 
paratus.

[15] M. L. Gorodetsky, A. A. Savchenko, and V. S. Ilchenko, Opt. Lett. 21, 453 (1996).

[16] R. E. Prange, R. Narevich and O. Zaitsev, Phys. Rev. E 59, 1694 (1999)
[17] S. M. Spillane, T. J. Kippenberg and K. J. Vahala, Nature 415, 621 (2002); and references therein.

[18] D. K. Armani, T. J. Kippenberg, S. M. Spillane and K. J. Vahala, Nature 421, 925 (2003) 